\title{
25 Research Square \\ Clinical characteristics of the lamellar macular hole according to two subtypes: a retrospective analysis
}

\section{Se Young Kim}

Inha University College of Medicine https://orcid.org/0000-0002-1005-5254

Myung Hun Yoon

Inha University College of Medicine

Hee Seung Chin ( $\nabla$ hschin@inha.ac.kr)

https://orcid.org/0000-0003-2104-3504

Research article

Keywords: Lamellar macular hole; Degenerative type; Tractional type;

Posted Date: May 9th, 2019

DOI: https://doi.org/10.21203/rs.2.9501/v1

License: (c) (i) This work is licensed under a Creative Commons Attribution 4.0 International License.

Read Full License 


\section{Abstract}

Background: The present study compared anatomical parameters and clinical characteristics of two subtypes of lamellar macular hole (LMH): tractional and degenerative. Methods: This retrospective chartreview study included patients who were monitored for more than 6 months after the initial diagnosis of LMH, from January 2011 to January 2018. Changes in the following morphological characteristics across the study period and between the two subtypes were assessed: the mean central foveal thickness, maximum outer retina diameter (MOD), and maximum inner retina diameter (MID). Differences in the following clinical parameters between the two conditions was also assessed: best corrected visual acuity (BCVA), anatomical progression rate, rate of surgery, and mean period before surgery. Results: This study included 51 eyes of 49 patients with a mean follow-up period of 18.94 months in the study: 33 tractional eyes and 18 degenerative eyes. The difference in the mean central foveal thickness between the two groups at baseline and the end of follow-up tended toward significance. MID was not significantly different between the two subtypes at either time point. MOD was significantly different between two subtypes at baseline and the end-of-follow up (tractional, $1131.62 \mu \mathrm{m}, 1358.18 \mu \mathrm{m}$; degenerative, 708.88 $\mu \mathrm{m}, 697.83 \mu \mathrm{m} ; \mathrm{p}<0.01 \mathrm{for}$ both). The changes in the retinal diameters across the observation period were significantly increased in degenerative eyes, and tractional eyes featured a significant increase in MOD across the study period. BCVA was not significantly different between the two subtypes at baseline and the last follow up. Anatomical progression rate of tractional eyes ( $81.8 \%$ ) was significantly higher than that of degenerative eyes $(27.7 \%)(p=0.01)$. The presence of ERM was significantly different between two subtypes (tractional, 96.9\%; degenerative, 22.2\%; $p<0.001$ ). Ellipsoid defect, rate of receiving surgery, and mean observation period before surgery were not significant different between the two subtypes. Conclusion: Analyzing two LMH subtypes according to their clinical and morphologic features, the present study identified characteristics unique to each. Treatment of LMH patients will require different approaches based on the subtype exhibited by the patient.

\section{Background}

In 1975, Gass $^{1}$ described a surface macular lesion of the multilobulated cystic macular edema as a lamellar macular hole (LMH) diagnosable with conventional biomicroscopy. Because the microscopyinformed diagnosis was ambiguous, optical coherence tomography (OCT) was developed to improve examination of the internal structure of the retina and thereby to help improve diagnostic efficacy. ${ }^{2-4}$ OCT allows for the distinction of $\mathrm{LMH}$ from other macular diseases, such as pseudohole. ${ }^{5} \mathrm{LMH}$ is now defined as a partial-thickness defect of the macula with an irregular foveal outline and isolation between the outer and inner retinal layers. ${ }^{7}$ While prior research has characterized the histology of LMH as thinning of the foveal tissue, separation of the RPE, and preservation of the photoreceptor layers with partial loss of the inner nuclear layer, the pathogenic mechanism of LMH has yet to be elucidated clearly. ${ }^{6,7}$

The development of OCT revealed LMH to be highly heterogeneous in morphology and function. Epiretinal proliferation occurs in approximately $30 \%$ of LMH cases and differs from formal epiretinal 
membranes (ERMs). Without any has no evidence of traction or retinal folds, this proliferation appears dense and yellow, further distinguishing it from formal ERMs; these findings may indicate that the proliferation does not include myoblasts.

Previous studies have classified LMH into two subtypes through anatomical OCT imaging findings: (1) tractional, which is thought to be caused by horizontal forces exerted by ERM and vitreomacular traction (VMT); (2) degenerative, which is clearly distinguished by a lack of a traction element despite the ambiguity in its underlying mechanism. Redefining and characterizing LMH as two apparently distinct clinical entities will help to advance understanding of the pathophysiology LMH and informed more specific, target treatments of the condition. ${ }^{7}$ Toward this end, the present study compared anatomical parameters and clinical characteristic parameters of LMH according to the two aforementioned subtypes.

\section{Methods}

This study adhered to the Helsinki Declaration and received approval from the institutional review board of Inha University Hospital. We reviewed the medical records of patients who were monitored for more than 6 months after being diagnosed with LMH at Inha University Hospital Eye Center (Incheon, Korea) from January 2011 to January 2018.

Patients were excluded from the study if they had exhibited any of the following: history of retina surgery, diabetic retinopathy, retinal vein occlusion, age related macular degeneration, choroidal neovascularization, and uveitis.

To perform spectral domain optical coherence tomography (SD-OCT), Cirrus HD-OCT (version 6.0 software) was used in all cases.

Morphologic parameters were defined as mean central foveal thickness, maximum inner/outer diameter (MID/MOD), presence of epiretinal membrane, and defects in the ellipsoid zone.

The clinical parameters included best corrected visual acuity, anatomical progression rate, rate of surgery, and mean period before surgery.

The mean central foveal thickness was measured as the average thickness within $500 \mu \mathrm{m}$ from the macular center. The anatomical progression was defined as an increase of $>50 \mu \mathrm{m}$ in the inner or outer diameters from baseline. The MID was measured as the longest length of the ILM level using the caliper function of the Cirrus OCT, and the MOD was measured as the longest length in the intraretinal cavity.

Statistical analysis was performed using SPSS software version 18 (SPSS Inc., Chicago, IL, USA). Comparisons between the parameters of LMH subtypes were performed using the Kruskal-Wallis test. The Wilcoxon signed rank test was performed to assess changes in parameters from baseline to the end of follow-up. Statistical significance was considered set to $p<0.05$. 


\section{Results}

Of the 80 eyes treated during the study period, 51 eyes of 49 patients ( 15 men; mean age, $66.37 \pm 10.79$; age range, 37 to 82 years; mean follow-up time; $18.94 \pm 16.64$ months) met the inclusion criteria and were enrolled in the study; 18 and 33 eyes were included in the degenerative and tractional groups, respectively. The mean ages of the patients in the tractional degenerative groups were similar: $65.32 \pm 11.72$ years and $68.72 \pm 8.18$, respectively ( $p=0.49$ ). No significant difference was found between the mean followup periods between the tractional and degenerative groups: $16.57 \pm 12.52$ months and $23.27 \pm 22.11$, respectively $(p=0.81)$. Sex ratios were significantly different between the two groups: the tractional group featured more women than did the degenerative group $(p<0.01)$. The 29 eyes either met the exclusion criteria or had been subjected to a different OCT analysis.

Tractional eyes exhibited a thicker mean central foveal thickness than degenerative eyes at the last follow up: $324.61 \pm 58.52 \mu \mathrm{m}$ vs $300.88 \pm 70.88 \mu \mathrm{m}$. This difference tended towards statistical significance $(\mathrm{p}=$ 0.09). Mean central foveal thickness did not increase significantly across the follow-up period in either group (tractional type, $319.35 \pm 52.61$ at baseline vs $324.61 \pm 58.52$ at the last follow-up examination, $p=$ 0.508 ; degenerative type, $300.00 \pm 64.82$ at baseline vs $300.88 \pm 70.88$ at the last follow-up examination, $p=0.931)$

The progressions of the mean inner and outer diameters differed according to subtype. In the tractional type, while the mean inner diameter had not changed significantly by the last follow-up $(p=0.338)$, the mean outer diameter increased significantly across the follow-up period $(p=0.003)$. In the degenerative type, the mean outer diameter did not change significantly across the follow-up period $(p=0.231)$, but the mean inner diameter had significantly increased by the last follow-up $(p=0.045)$. The mean outer diameter of tractional eyes was significantly larger than that of degenerative eyes at baseline and the lase of follow-up ( $p<0.01$ for both).

The best corrected visual acuity did not change across follow up in either group (tractional type, 0.224 $\log M A R, 0.236 \log M A R, p=0.19$; degenerative type, $0.147 \log M A R, 0.156 \log M A R ; p=0.09$ ).

The inner/outer diameter ratio was smaller and the ERM was more commonly observed in the tractional group than in the degenerative group (MID/MOD ratio < 1:2: tractional type, 63.6\%; degenerative type, $11.1 \% ; p<0.001$; ERM: tractional type, 96.9\%; degenerative type, $22.2 \% ; p<0.001)$. In the degenerative group, although not statistically significant, ellipsoid zone defect was more frequently observed (tractional type, 27.2\%; degenerative type, 38.8\%; $p=0.24$ ).

The anatomical progression rate was much higher in the tractional group than in the degenerative group (tractional type, $81.8 \%$; degenerative type, $27.7 \% ; p=0.01$ ). Rate of macular surgery was not significantly different between the groups but was higher in the tractional group than in the degenerative group (tractional type, 30.3\%; degenerative type, $22.2 \% ; p=0.58$ ). 


\section{Discussion}

The pathogenesis of LMH remains unclear. The development of OCT allowed for high resolution observation of morphologic features characteristic of LMH; these have been document by several studies, ${ }^{8}$ and several redefinitions of LMH have been proposed. Time-domain OCT investigation have explained LMH morphologic features in terms of the irregular foveal contour, separation of foveal edges, and standard perifoveal thickness. ${ }^{9}$ Spectral-domain OCT studies have described the morphologic features of LMH in four ways: irregular foveal contour, break in the inner fovea, intraretinal split situated between the outer plexiform and nuclear layers, and intact foveal photoreceptors. ${ }^{12}$ However, these LMH concepts have not unified the various morphologic and clinical observations.

It is particularly difficult to ascribe intraretinal cyst caused by tractional force to the concept of LMH: though several concepts have been proposed to explain these cases, none could explain the LMH to unify and prognosis of the data of these definitions were observed differently. The controversy among current definitions of $\mathrm{LMH}$ indicates the need for its redefinition.

Thus, Govetto et al classified LMH into two subtypes: tractional and degenerative. The former is defined by the presence of traction, such as ERM and VMT, while the latter is exhibits traction-free epiretinal proliferation. $^{7}$

In tractional LMH, horizontal ERM- and VMT-induced tractional forces affect the outer diameter rather than the inner diameter. Indeed, the present study revealed that tractional eyes exhibited larger maximum outer diameters than did the degenerative eyes across the study period.

Thus, the rate of increase in the ratio between the inner and outer diameters is larger among tractional than degenerative eyes. In the case of the former, since the horizontal force is constant, the outer diameter increases by the end-of-follow-up compared to the baseline; hence, tractional eyes featured a relatively heightened anatomical progression rate. Our study also showed that the outer diameter increased during the follow-up period among tractional eyes. Previous studies demonstrated that observation alone achieved better clinical outcomes than did surgery, despite anatomic progression.

The conditions of degenerative eyes progresses gradually and chronically; many cases are only discovered accidentally during regular checkup, while others are based on the subject interpretation of symptoms. Tractional eyes often exhibit subjective symptoms such as deformity. For this reason, the best corrected visual acuity was superior in degenerative eyes, although the difference was not statistically significant.

In the case of patients tractional rapid hole size progression, the surgical procedure was performed to remove traction. The rate of surgery was therefore higher in the tractional group than in the degenerative group; however, this difference was statistically nonsignificant. 
This study is subject to the limitation of diminished statistical validity due to a small sample size. Because visual function is preserved in cases of $L M H$, there is a bias against completion of long-term follow-up observation. Moreover, could be easily classified as either tractional or degenerative; some cases presented features of both. ${ }^{7}$ Only best corrected visual acuity was used to assessed visual acuity. In future studies, it may be necessary to evaluate the quality of visual acuity in multiple aspects such as comparing metamorphosis between two subgroups. In addition, such additional metrics may help to indicate surgery.

In conclusion, this study analyzed morphologic and clinical features of two LMH subtypes and confirmed that both are characterized by different features: Tractional LMH features schitic separation of the outer segmentation retina. This entity is mostly associated with tractional ERM or VMT and thus accounts for the relatively quicker o anatomical progression in tractional eyes. Degenerative $\mathrm{LMH}$ is characterized by an intraretinal cavitation of all retinal layers. These lesions are the result of nontractional epiretinal proliferation caused by chronic, degenerative pathophysiology.

This report investigated the anatomic and clinical features characteristic to current classifications of $\mathrm{LMH}$. Our results will help to inform understanding of the characteristics and developmental mechanism underlying $\mathrm{LMH}$ pathology and consequently the treatment of patients with $\mathrm{LMH}$. The prognoses and treatments of patients with LMH may thus differ according on their LMH subtype.

\section{Abbreviations}

$\mathrm{LMH}$, lamellar macular hole; MOD, maximum outer retina diameter; MID, maximum inner retina diameter; BVBA, best corrected visual acuity; ERM, epiretinal membranes; VMT, vitreomacular traction.

\section{Declarations}

\section{Ethics approval and consent to participate}

This study was approved by the Ethics Committee of the Inha hospital, and conformed to the tenets of the

Declaration of Helsinki.

\section{Consent for publication}

Not applicable.

\section{Availability of data and material}

The datasets used and/or analysed during the current study available from the corresponding author on reasonable request. 


\section{Competing interests}

The authors declare that they have no competing intersets

\section{Funding}

No funding to declare

\section{Authors' contributions}

KSY analyzed and interpreted the patient data, and was a major contributor in writing the manuscript. $\mathrm{YMH}$

and $\mathrm{CHS}$ revised this paper. All authors read and approved the final manuscript.

\section{Acknowledgements}

None.

\section{References}

1 Gass JD, Norton EW. Lamellar macular hole: a complication of cystoid macular edema after cataract extraction. Arch Ophthalmol. 1976;94:793-800.

2 Gaudric A, Haouchine B, Massin P, Paques M, Blain P, ErginayA.Macular hole formation: new data provided by optical coherence tomography. Arch Ophthalmol. 1999;117:744-51.

3 Takahashi H, Kishi S. Tomographic features of a lamellar macular hole formation and a lamellar hole that progressed to a full-thickness macular hole. Am J Ophthalmol. 2000;130:677-9.

4 Haouchine B, Massin P, Gaudric A. Foveal pseudocyst as the first step in macular hole formation: a prospective study by optical coherence tomography. Ophthalmology .2001;108:15-22.

5 Haouchine B, Massin P. Diagnosis of macular pseudoholes and lamellar macular holes by optical coherence tomography. Am J Ophthalmol, 2004;138:732-9 
6 Okada M, Ogino N, Matsumura M. Histological and immunohistochemical study of idiopathic epiretinal membrane. Ophthalmic Res. 1995;27:118 -28.

7 ANDREA GOVETTO, YANN DACQUAY, MATTHEW FARAJZADEH et al. Lamellar Macular Hole: Two Distinct Clinical Entities? Am J Ophthalmol. 2016;164:99-109.

8 Androudi S, Stangos A, Brazitikos PD. Lamellar macular holes: tomographic features and surgical outcome. Am J Ophthalmol. 2009;148(3):420-6.

9 Pang CE, Spaide RF, Freund BK. Epiretinal proliferation seen in association with lamellar macular holes: a distinct clinical entity. Retina 2014;34(8):1513-23.

10 Compera D, Entchev E, Haritoglou Cl. Lamellar hole associated epiretinal proliferation in comparison to epiretinal membranes of macular pseudoholes. Am J Ophthalmol. 2015; 160(2):373-84.

11 Pang CE, Spaide RF, Freund BK. Comparing functional and morphologic characteristics of lamellar macular holes with and without lamellar hole-associated epiretinal proliferation. Retina 2015;35(4):7206.

12 Witkin AJ, Ko TH, Fujimoto JG. Redefining lamellar holes and the vitreomacular interface: an ultrahigh-resolution optical coherence tomography study. Ophthalmology. 2006;113:388-97

\section{Tables}


Table 1. Patient demographics

\begin{tabular}{cccc}
\hline & Total $(\mathrm{n}=51)$ & Degenerative group $(\mathrm{n}=18)$ & Tractional group \\
& & & $(\mathrm{n}=33)$ \\
\hline Sex & Male 15 $(29.4 \%)$ & Male 11 $(61.1 \%)$ & Male $4(12.1 \%)$ \\
& & & \\
Female 36 (70.6\%) & Female 7 (38.9\%) & Female 29 (87.9\%) \\
Age & $66.37 \pm 10.79$ years & $68.72 \pm 8.18$ years & $65.32 \pm 11.72$ years \\
\hline Mean f/u period 18.94 \pm 16.64 Months & $23.27 \pm 22.11$ Months & $16.57 \pm 12.52$ Months
\end{tabular}

Table 2. Anatomic and Clinical Characteristics of Degenerative and Tractional LMH

\begin{tabular}{|c|c|c|c|c|}
\hline Measurements & & egenerative gro & Tractional grou & \\
\hline \multirow[t]{3}{*}{ Mean central foveal thickness } & Baseline & $300.00 \mu \mathrm{m}$ & $319.35 \mu \mathrm{m}$ & $\mathrm{p}=0.14$ \\
\hline & End of follow-up & $300.88 \mu \mathrm{m}$ & $324.61 \mu \mathrm{m}$ & $\mathrm{p}=0.09$ \\
\hline & & $\mathrm{p}=0.931$ & $\mathrm{p}=0.508$ & \\
\hline \multirow[t]{3}{*}{ Maximum inner diameter } & Baseline & $654.22 \mu \mathrm{m}$ & $572.70 \mu \mathrm{m}$ & $\mathrm{p}=0.24$ \\
\hline & End of follow-up & $730.55 \mu \mathrm{m}$ & $602.88 \mu \mathrm{m}$ & $\mathrm{p}=0.07$ \\
\hline & & $\mathrm{p}=0.045$ & $\mathrm{p}=0.338$ & \\
\hline \multirow[t]{3}{*}{ Maximum outer diameter } & Baseline & $708.88 \mu \mathrm{m}$ & $1131.62 \mu \mathrm{m}$ & $\mathrm{P}<0.01$ \\
\hline & End of follow-up & $697.83 \mu \mathrm{m}$ & $1358.18 \mu \mathrm{m}$ & $\mathrm{P}<0.01$ \\
\hline & & $\mathrm{p}=0.231$ & $\mathrm{p}=0.003$ & \\
\hline \multirow[t]{3}{*}{ Best corrected visual acuity } & Baseline & 0.147logMAR & $0.224 \log \mathrm{MAR}$ & $\mathrm{p}=0.19$ \\
\hline & End of follow-up & 0.156logMAR & $0.239 \log \mathrm{MAR}$ & $\mathrm{p}=0.09$ \\
\hline & & $\mathrm{p}=0.759$ & $\mathrm{p}=0.532$ & \\
\hline
\end{tabular}


Table 3. Anatomic and Clinical Characteristics of Degenerative and Tractional LMH

\begin{tabular}{cccc}
\hline & Degenerative group & Tractional group & $\mathrm{p}$-value \\
\hline $\begin{array}{c}\text { Inner/outer diameter } \\
\text { ratio }<1: 2\end{array}$ & $11.1 \%$ & $63.6 \%$ & $\mathrm{P}<0.001$ \\
Epiretinal membrane & $22.2 \%$ & $96.9 \%$ & $\mathrm{p}<0.001$ \\
Ellipsoid defect & $38.8 \%$ & $27.2 \%$ & $\mathrm{P}=0.24$ \\
& & & \\
Anatomical progression & $27.7 \%$ & $81.8 \%$ & $\mathrm{P}=0.01$ \\
\hline Rate of receiving surgery & $22.2 \%$ & $30.3 \%$ & $\mathrm{P}=0.58$ \\
& & & $\mathrm{p}=0.50$ \\
Mean period & 6.0 months & 12.5 months & \\
\hline before surgery & & &
\end{tabular}

\section{Figures}




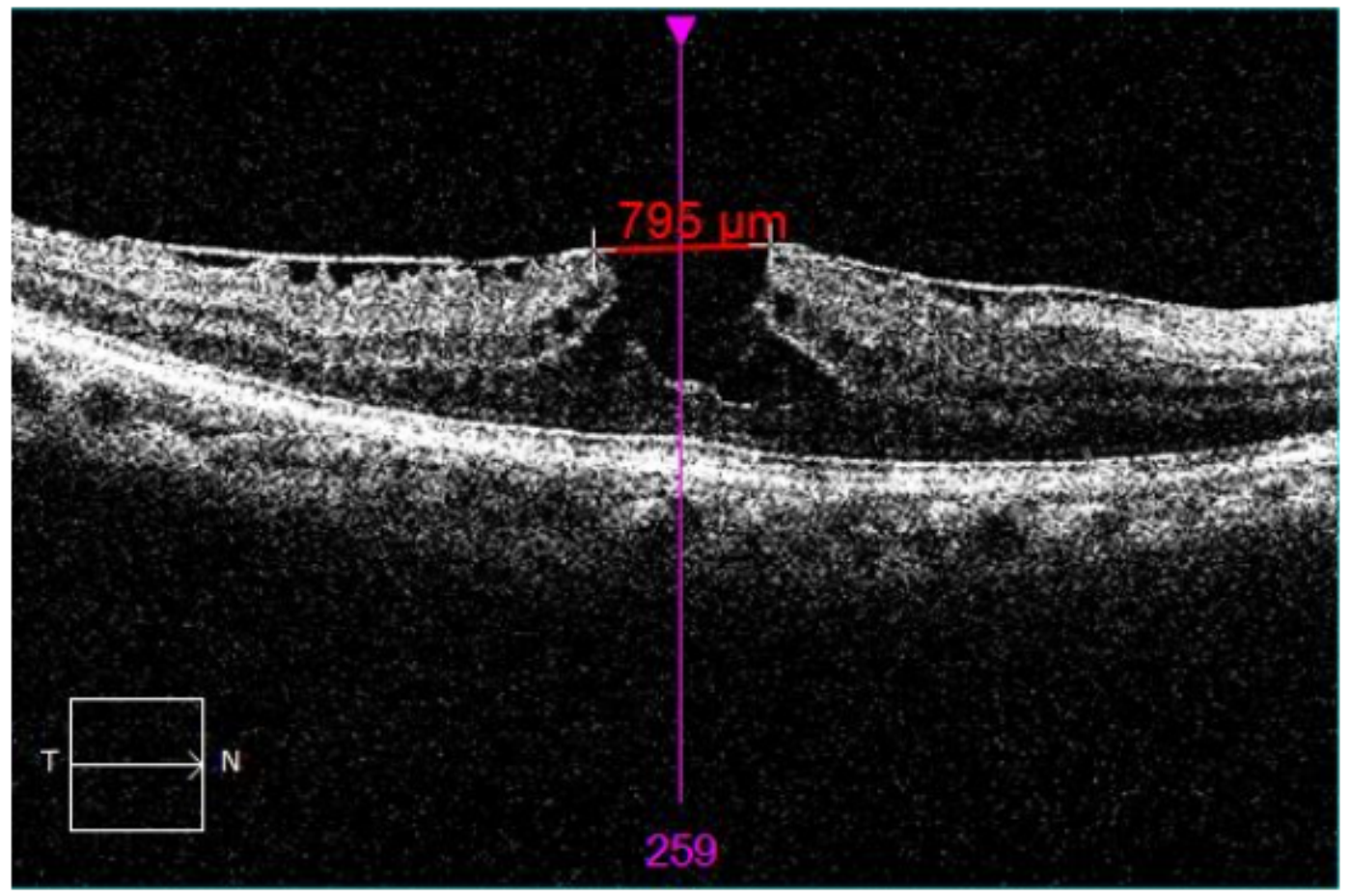

(B) Maximum outer diameter

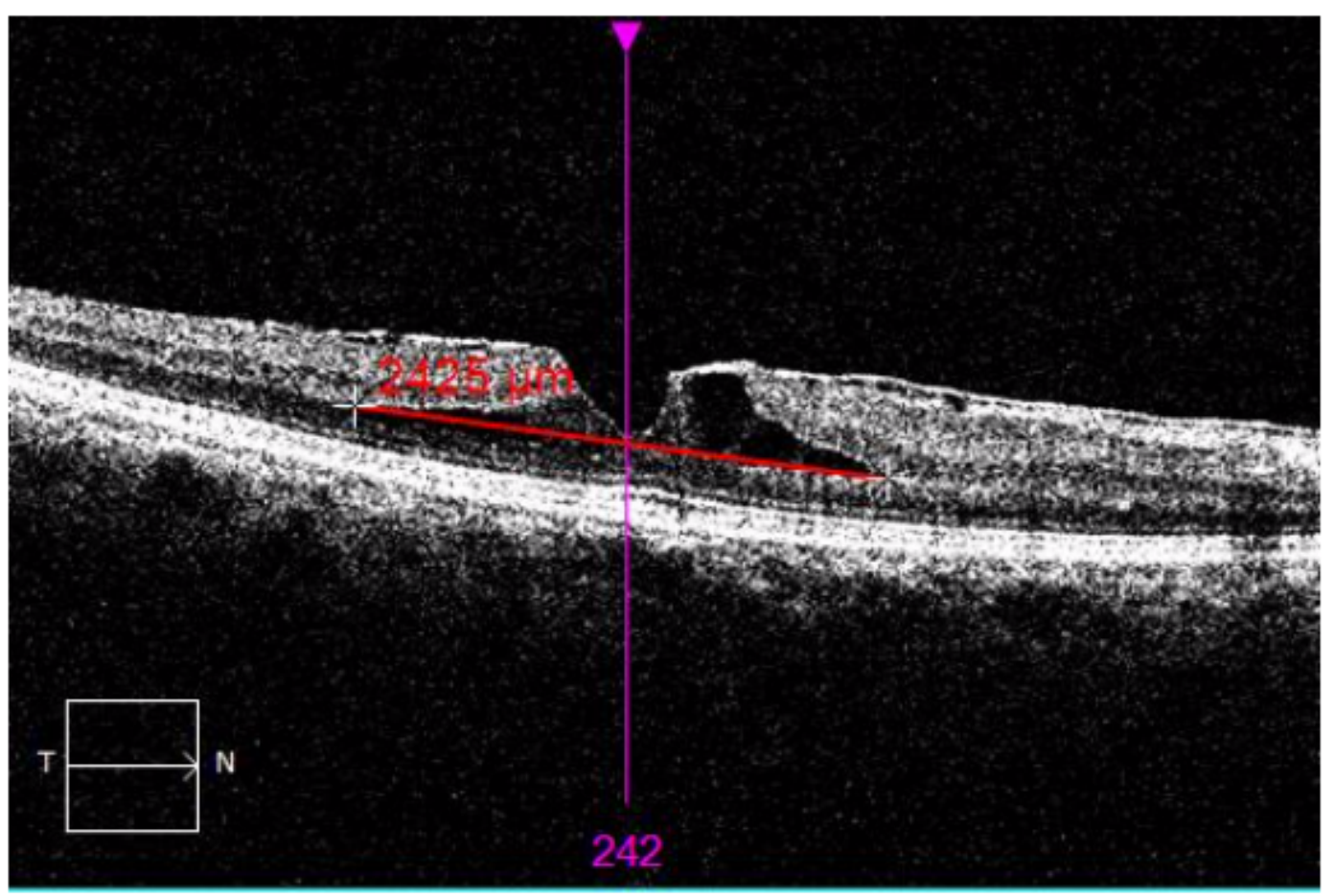

\section{Figure 1}

The measurement of the retinal diameter through the caliper of optical ocherence tomography software is described. 